Journal of Animal and Veterinary Advances 11 (17): 3223-3227, 2012

ISSN: $1680-5593$

(C) Medwell Journals, 2012

\title{
Sequencing of the Cattle Genome Toward Finding Ways to Increase Feed Efficiency of Cattle
}

\author{
Woonsu Kim and Seongwon Seo \\ Department of Animal Biosystem Sciences, Chungnam National University, \\ 305-764 Daejeon, Republic of Korea
}

\begin{abstract}
Considering the continuous increase in feed cost, the greatest part of the cattle production cost and the growth of environmental concerns, improvement of feed efficiency has potentials not only for increasing profits for cattle producers but also for decreasing environmental impacts of cattle production. The recent sequencing of the cattle genome provides the first opportunity to explore the relationship between genetic and feed efficiency of cattle. This short review aims to describe recent findings from the sequencing analysis of the cattle genome and to illustrate how this new information can be applied increase feed efficiency of cattle.
\end{abstract}

Key words: Cattle, feed cost, production, efficiency, growth, profit

\section{INTRODUCTION}

Now a days the cattle industry needs to consider two big things profitability and environmental impacts. The cattle industry is a business and thus it should maximize its profitability. As profit is calculated as income minus cost the cattle industry is elaborating upon a plan to reduce the production cost and to increase the amount and value of products. Since, the cost related feed is the major portion of the production cost reducing feed cost would be one of the most effective ways to increase profitability. However, this is quite challenging because the average price of feed ingredients has been increasing dramatically. For instance, the average price of the major feed ingredients (e.g., corn and soybean meal) became doubled in 2007 compared to 2001 .

Another big challenge that the beef industry encounters is that the animal industry is under pressure with minimizing environmental impacts as environmental concerns grow globally. One of the pollutants of great concern is greenhouse gas emissions from cattle. A recent statistics showed that methane productions from enteric fermentation by beef and dairy cattle were estimated to be 100.2 and $31.9 \mathrm{Tg} \mathrm{CO}_{2}$ equivalent which contributed 53 and $17 \%$ of the methane emissions from the agriculture sector in the US (EPA, 2009). Nitrogen and phosphorus losses from cattle are also possible negative impacts of the cattle industry to the environment. Methane production and nitrogen and phosphorus losses not only contribute environmental pollutions but also represent losses of nutrient that can be used for production otherwise.

Two challenges look different at the first sight. However, if we look at them closely reserchers can easily find out that both issues are related with the feed consumed and utilized by cattle. In other words, efficient use of feed nutrients would decrease the amount of feed needed for supporting the growth of cattle and reduce nutrients losses to the environment. Thus, there has been a strong rationale for developing genomic resources and nutrition plans that can be used to increase feed efficiency of cattle.

In this regards, the recent sequencing of the cattle genome (Elsik et al., 2009) provides the first opportunity to explore the relationship between genetic and important economic traits of cattle including feed efficiency. Sequencing and analysis of the cattle genome enabled a genome-scale reconstruction of metabolic pathways in the cattle genome which can be used to analyze interpret and predict the genotype-to-phenotype relationships in an organism (Schilling et al., 1999). Genomics, together with bioinformatics and systems biology is expected to lead to revolutionary changes of the studies in the animal science field (Hocquette et al., 2007).

This short review aims to describe recent findings from sequencing and analyzing the cattle genome and to illustrate how this new information can be applied increase feed efficiency of cattle.

Corresponding Author: Seongwon Seo, Department of Animal Biosystem Sciences, Chungnam National University, 305-764 Daejeon, Republic of Korea 


\section{GENOME SEQUENCING OF THE CATTLE GENOME}

Sequencing of the cattle genome started in 2003 lead by the Baylor College of Medicine Sequencing Center in Houston, TX, USA. Researchers from a total of 25 countries developed a consortium and have collaboratively worked on sequencing the genome of a Hereford cow named L1 Dominette 0144. The genome was sequenced to $\sim 7 \mathrm{x}$ coverage using a combined whole genome shotgun and BAC skim approach and the fourth draft (Btau_4.0) was assembled and a reference gene set was annotated (Elisk et al., 2009). In the current build (Btau_4.0) chromosomes cover about 89\% of the total genome and $95 \%$ of the 1.04 million Expressed Sequence Tag (EST) sequences are contained in the assembled contigs.

The size of the cattle genome was estimated to be $2.87 \mathrm{Gbp}(2.73 \mathrm{Gbp} / 0.95)$ assuming the ESTs are uniformly distributed throughout the genome. The alignment of 73 finished Bacterial Artificial Chromosome (BAC) to the assembly showed the quality of the assembly is high with a coverage $98.5 \%$ of the $\mathrm{BAC}$ sequences aligned to the assembly.

\section{ANALYSIS OF THE CATTLE GENOME}

Using a computational tool, GLEAN (Elsik et al., 2007), a consensus set of 26,835 $a b$ initio genes were annotated. Using RT-PCR on RNA collected from 12 bovine tissues, $82 \%$ of the GLEAN genes were experimentally validated. It was thus estimated that the bovine genome contains a minimum of 22,000 genes $(82 \%$ of 26,835$)$.

One of the interesting findings from the genome analysis is that the protein-coding genes of cattle are more conserved and similar to those of human than mouse and rat. Overall high levels of gene and protein conservation between cattle and human were observed (Fig. 1a). A genome-wide maximum-likelihood phylogenetic analysis based on single-copy orthologs showed the cattle lineage was diverged from human lineage earlier than the mouse lineage which was consistent with the commonly accepted phylogeny (Murphy et al., 2007).

However, the level of sequence identity between human and cattle proteins was higher than between human and mouse. These results suggest a promising of cattle as an animal model for human studies.
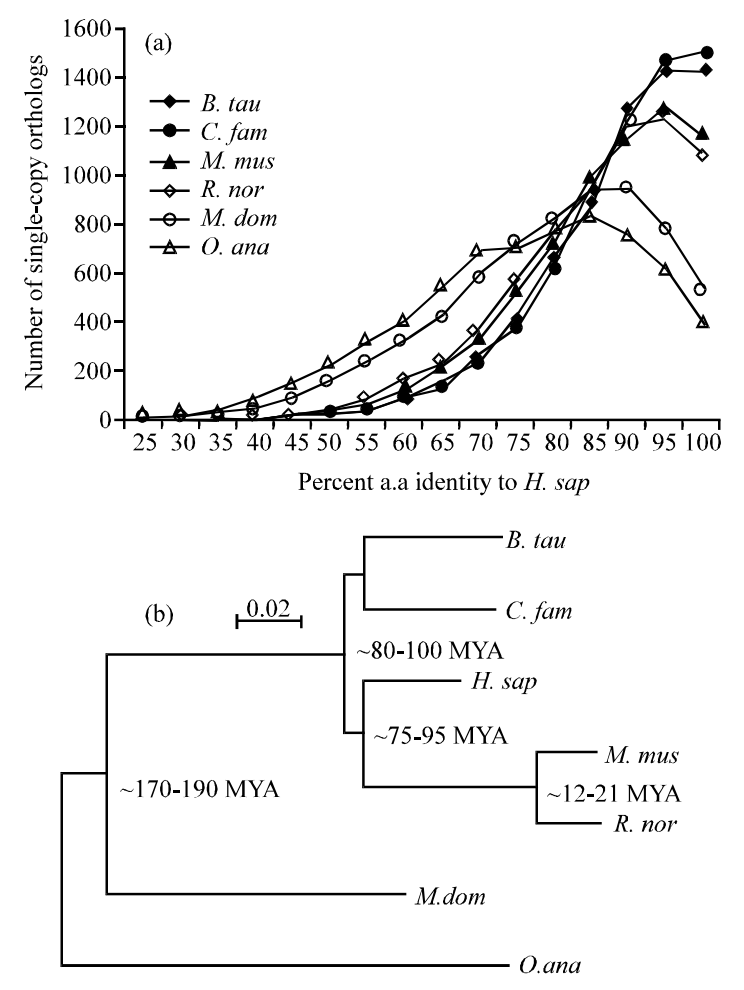

Fig. 1: Protein orthology comparison among genomes of cattle, dog, human, mouse and rat (Bos taurus, Canis familiaris, Homo sapiens, Mus musculus Rattus norvegicus, representing placental mammals), opossum (Monodelphis domestica, marsupial) and platypus (Ornithorhynchus anatinus, monotreme). Modified from Elisk et al. (2009). a) Distribution of orthologs protein identities between human and the other species for a subset of strictly conserved single-copy orthologs. b) A maximum likelihood phylogenetic tree using all single-copy orthologs supports the accepted phylogeny and quantifies the relative rates of molecular evolution expressed as the branch lengths

\section{RECONSTRUCTION OF METABOLIC PATHWAYS OF THE CATTLE GENOME}

Using bioinformatic tools followed by comprehensive manual curation, the metabolic pathways encoded in the cattle genome has been reconstructed (Seo and Lewin, 2009). After a genome is sequenced, metabolic reconstruction of the sequenced genome is a necessary step toward understanding the metabolism and underlying mechanism of metabolic regulation (Reed et al., 2006). The reconstructed cattle metabolic 


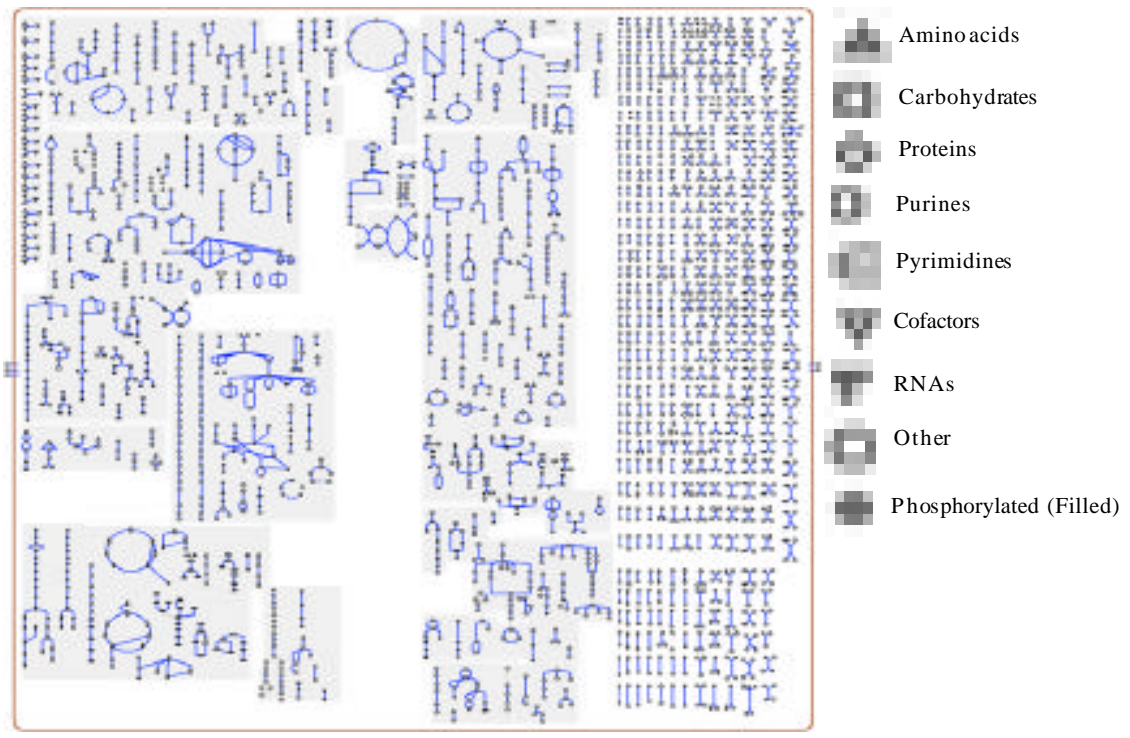

Fig. 2: Reconstructed metabolic pathways of the cattle genome based on Btau_3.1. The figure was generated by the cellular overview tool at cattlecyc (http://biocyc.org/CATTLE/)

pathways consist of 217 metabolic pathways, 1,544 enzymes, 1,442 biochemical reactions and 1,021 compounds (Fig. 2).

Reconstructed metabolic pathways of the cattle genome revealed a strong degree of conservation among the comprehensive set of genes involved in core cellular metabolism (Seo and Lewin, 2009). The complete metabolic reconstruction of the cattle genome also permits an examination of unique genetic events that may be related to ruminant-specific metabolic adaptations. Comprehensive bioinformatic analyses proved that among 1,032 genes in reconstructed human metabolic pathways five genes are deleted or extensively diverged in the cattle genome: PLA2G4C, FAAH2, IDI2 and GSTT2 and TYMP. None of these genes have EST or mRNA evidence and all are completely absent from well-assembled sequence or show signs of extensive divergence on the basis of multiple sequence alignments. Deletions of these genes may imply cattle-specific adaptations in phospholipid metabolism fatty acid metabolism the mevalonate pathway (synthesis of prenylated proteins, dolichols, Vitamins A, $\mathrm{D}, \mathrm{E}$ and $\mathrm{K}$, steroid hormones, carotenoids, bile acids and cholesterol), detoxification and pyrimidine metabolism, respectively.

Comparative analysis for identification of genetic differences between animal species and within a species is very important because genetic differences are related with divergent metabolic traits and thus they could be used as biomarkers for Marker Assisted Selection (MAS).

\section{APPLICATIONS OF GENOMIC INFORMATION TO INCREASE FEED EFFICIENCY OF CATTLE}

Traditionally feed efficiency has been defined as a ratio of feed:gain or feed conversion ratio however, they are undesirable because of their correlation with growth rate or BW (Koots et al., 1994). Thus, Residual Feed Intake (RFI) defined as the difference between actual and predictd feed intake relative to weight and ADG (Koch et al., 1963) has become widely used to measure feed efficiency in beef cattle (Moore et al., 2009). RFI is independent of body weight and $\mathrm{ADG}$ and it is moderately heritable with a range from 0.28-0.58 (Moore et al., 2009). Many studies support that animal selection for RFI is possible and reduced feed intake can be heritable to offspring (Moore et al., 2009).

Since, experimental measurement of RFI is expensive and time-consuming there have been attempts to develop genetic and molecular tools to measure RFI indirectly and to identify bio-markers (indicators) for genetic potential with a negative RFI. The level of Insulin-like Growth Factor-1 (IGF-1) in plasma was thought to be a good indicator of increased feed efficiency (Moore et al., 2005). However, the reliability of this physiological maker has been recently challenged due to inconsistent results (Herd and Arthur, 2009).

Recent advances in genomics are encouraging considerable research that determines the genetic basis of RFI, especially for finding Quantitative Trait Loci (QTL). So far only a limited number of studies 
have been published (Moore et al., 2009). Using 10,000 Single-Nucleotide Polymorphisms (SNPs), Barendse et al. (2007) identified 161 SNPs that were significantly associated with RFI. Among these, 20 SNPs explained $76 \%$ of the amount of the genetic variance for RFI and 90 contained micro-RNA motifs and 86 contained promoter elements in the sequence flanking the SNPs which imply that nongenic variants are important in controlling feed efficiency. They also found that a greater number of genes involved in the energetic pathways including cell growth and development are located at the flanking of the SNPs (Barendse et al., 2007).

Nkrumah et al. (2007) also did a primary genome scan to identify putative QTL for RFI. The markers used were 100 microsatellites and 355 SNPs on the cattle autosomes (Nkrumah et al., 2007). They found 8 QTLs for RFI on chromosomes $1,5,7,8,12,16,17$ and 26 with a chromosome-wise $\mathrm{p}<0.05$.

Cattle genome sequencing enabled identification of 37,470 SNPS in 497 cattle from 19 geographically and biologically diverse breeds (Gibbs et al., 2009). During the research it was found that SNP (rs29021800) with flanking genes of ZRANB3 (Zinc finger, RAN-binding domain containing 3) and R3HDM1 (R3H domain containing 1) located on chromosome 2 between 64,740,286 and $64,931,017$ is associated with feed efficiency. This region is also associated with intramuscular fat content in some breeds (Gibbs et al., 2009). The recent development of 60K SNP chip (Van Tassell et al., 2008) will facilitate fine-mapping of QTLs for feed efficiency.

\section{CONCLUSION}

Improvement of feed efficiency of cattle could both maximize the profits and minimize negative environmental impacts of the cattle industry. The recent sequencing of the cattle genome provides tremendous amount of genomic resources for accessing the genotype and phenotype relationships which can be used to increase feed efficiency of cattle. Although, there are a limited number of the published genome-wide association studies, the number will be increased dramatically in near future. Application of recent omics technology and systems biology may be helpful to decipher and understand the gene and protein networks that are related with feed efficiency and it will accelerate development of genetic and nutritional programs that can be practically applied to increase feed efficiency of cattle in the field.

\section{ACKNOWLEDGEMENTS}

This research was supported by a Basic Science Research Program (No. 2009-0064205) through National
Research Foundation of Korea funded by the Ministry of Education, Science and Technology and by a grant from the Next-Generation BioGreen 21 program (No. PJ008191) rural development Administration, Republic of Korea.

\section{REFERENCES}

Barendse, W., A. Reverter, R.J. Bunch, B.E. Harrison, W. Barris and M.B. Thomas, 2007. A validated whole-genome association study of efficient food conversion in cattle. Genetics, 176: 1893-1905.

EPA, 2009. Inventory of U.S. greenhouse gas emissions and sinks: 1990-2007. U.S. Environmental Protection Agency, Washington, DC, USA.

Elsik, C.G., A.J. Mackey, J.T. Reese, N.V. Milshina, D.S. Roos and G.M. Weinstock, 2007. Creating a honey bee consensus gene set. Genome Biol., 8: R13-R13.

Elsik, C.G., R. Gibbs, L. Skow and R. Tellam et al., 2009. The genome sequence of taurine cattle: A window to ruminant biology and evolution. Science, 324: $522-528$.

Gibbs, R., C.P. Van Tassell, G.M. Weinstock and R.D. Green et al., 2009. Genome-Wide survey of SNP variation uncovers the genetic structure of cattle breeds. Science, 324: 528-532.

Herd, R.M. and P.F. Arthur, 2009. Physiological basis for residual feed intake. J. Anim. Sci., 87: E64-E71.

Hocquette, J.F., S. Lehnert, W. Barendse, I. Cassar-Malek and B. Picard, 2007. Recent advances in cattle functional genomics and their application to beef quality. Animal, 1: 159-173.

Koch, R.M., L.A. Swiger, D. Chambers and K.E. Gregory, 1963. Efficiency of feed use in beef cattle. J. Anim. Sci., 22: 486-494.

Koots, K.R., J.P. Gibson and J.W. Wilton, 1994. Analyses of published genetic parameter estimates for beef production traits. 2. Phenotypic genet correlations. Anim. Breed Abstr., 62: 825-853.

Moore, L.L., D.J. Johnston, H.U. Graser and R.M. Herd, 2005. Genetic and phenotypic relationships between insulin-like growth factor-I (IGF-I) and net feed intake, fat and growth traits in Angus beef cattle. Aust. J. Exp. Agr., 56: 211-218.

Moore, S.S., F.D. Mujibi and E.L. Sherman, 2009. Molecular basis for residual feed intake in beef cattle. J. Anim. Sci., 87: 41-47.

Murphy, W.J., T.H. Pringle, T.A. Crider, M.S. Springer and $\mathrm{W}$. Miller, 2007. Using genomic data to unravel the root of the placental mammal phylogeny. Genome Res., 17: 413-421.

Nkrumah, J.D., E.L. Sherman, C. Li, E. Marques and D.H. Crews et al., 2007. Primary genome scan to identify putative quantitative trait loci for feedlot growth rate, feed intake and feed efficiency of beef cattle. J. Anim. Sci., 85: 3170-3181. 
Reed, J.L., I. Famili, I. Thiele and B.O. Palsson, 2006. Towards multidimensional genome annotation. Nat. Rev. Genet., 7: 130-141.

Schilling, C.H., S. Schuster, B.O. Palsson and R. Heinrich, 1999. Metabolic pathway analysis: Basic concepts and scientific applications in the post-genomic era. Biotechnol. Prog., 15: 296-303.
Seo, S. and H.A. Lewin, 2009. Reconstruction of metabolic pathways for the cattle genome. BMC Syst. Biol., Vol. 3. 10.1186/1752-0509-3-33

Van Tassell, C.P., T.P. Smith, L.K. Matukumalli, J.F. Taylor and R.D. Schnabel et al., 2008. SNP discovery and allele frequency estimation by deep sequencing of reduced representation libraries. Nat. Methods, 5: $247-252$. 